\title{
THE MICROBIOLOGICAL DIAGNOSIS OF TUBERCULOSIS IN A RESOURCE - LIMITED SETTING: IS ACID-FAST BACILLI MICROSCOPY ALONE SUFFICIENT?
}

\author{
*M.O. Odubanjo, and ${ }^{* *}$ H.O. Dada-Adegbola
}

* Department of Pathology, University College Hospital, Ibadan.

**Department of Medical Microbiology and Parasitology, University College Hospital, Ibadan.

Correspondence:

Dr. M.O. Odubanjo

Department of Pathology,

University College Hospital,

Ibadan, Nigeria.

\begin{abstract}
The objective of this study is to audit the processes for the microbiological diagnosis of tuberculosis (TB) in our resource-limited setting. A total of 694 specimens were received from 333 patients. $129(38.7 \%)$ of these patients were positive for TB. $78(60.5 \%)$ were positive on AFB microscopy alone, $13(10.0 \%)$ on culture alone and $38(29.5 \%)$ on both culture and AFB microscopy. Fifty-one (51) cases were positive on culture, 38 of these (74.5\%) had growth on Lowensen-Jensen culture medium alone, 11 (19.6\%) on Pyruvic Acid Enhanced Medium (PAEM) and 3 (5.9\%) on both culture media. AFB microscopy showed a diagnostic specificity of $71.6 \%$ and a sensitivity of $74.5 \%$. M. Bovis appears to be prevalent and we suggest the need for speciation. If AFB microscopy is to be routinely used alone, without confirmation by culture, then the overriding need is for quality to be fully assured in its use.
\end{abstract}

Keywords: Mycobacteria, tuberculosis, accuracy, AFB microscopy, culture

\section{INTRODUCTION}

Tuberculosis has great political and economic importance both in Nigeria and in the world as a whole. The global burden of the disease is huge. ${ }^{1}$ The identification of infectious patients is one of the key aspects of tuberculosis control. This is because each individual with untreated smear positive tuberculosis infects 10-15 persons per year. ${ }^{2}$ A significant proportion of new cases of tuberculosis are sputum smear positive. WHO estimates show that close to $50 \%$ of new cases of tuberculosis in 2004 were sputum smear positive. $^{2}$

Based on the estimated number of incident cases of tuberculosis, Nigeria ranked fifth in the WHO Africa Region in 2006. ${ }^{3}$ The Tuberculosis Laboratory at the University College Hospital (UCH), the location of this study, has a pivotal role to play in ensuring qualityassured bacteriology as part of the WHO Stop TB Strategy ${ }^{4}$ in that it is one of the six (6) zonal reference laboratories for Tuberculosis in Nigeria. It serves the South-West zone of Nigeria. ${ }^{3}$

In Nigeria, while the case notification rate for tuberculosis has become satisfactory, the case detection rate, even within Directly Observed Treatment Short course (DOTS) areas, is still below the target of $70 \%{ }^{3,4}$, hence there is need for a study to audit the diagnostic methods most commonly used.

There are a number of studies from the $\mathrm{UCH}$, Ibadan and other centres in Nigeria describing the peculiarities of tuberculosis in various medical sub-specialties. However, there has been no previous study to audit the diagnostic service for tuberculosis at the $\mathrm{UCH}$.

In spite of new technologies ${ }^{5,6,7,8}$ for diagnosing tuberculosis, AFB microscopy and culture still constitute the mainstay of the diagnostic procedure for tuberculosis at the UCH. The Tuberculosis Laboratory at the UCH, Ibadan has the BACTEC460 and MGIT (Mycobacteria Growth Indicator Tube) broth media available with efforts being made to acquire facilities for the molecular diagnosis of tuberculosis.

The global strategy for the control of tuberculosis, DOTS identifies cases by AFB microscopy only, ${ }^{4,9}$ this has the advantage of early commencement of treatment. In view of this, it is imperative that AFB microscopy is studied, standardized and its specificity and sensitivity against a more accurate method such as culture determined.

Our aim was to audit the processes for diagnosing tuberculosis in our resource-limited setting with the aim of improving our services and ensuring that the best possible service is rendered to our tuberculosis patients. We hope that this would ultimately improve the case detection rate for tuberculosis at the $\mathrm{UCH}$ and other parts of Nigeria.

\section{MATERIALS AND METHODS}

This was a retrospective study that was carried out in October 2007 recruiting the most recent patients for 
whom both AFB microscopy and culture had been done, the specimens were examined within the period from January 1996 to December 1998, as routine mycobacterial culture was discontinued shortly after this period. The laboratory records of all suspected cases of tuberculosis for which requests had been received at the Tuberculosis Laboratory of the Department of Medical Microbiology and Parasitology of the UCH, Ibadan were retrieved.

\section{Patient selection}

We recruited into the study only those patients who fulfilled the 2006 WHO requirements for the diagnosis of tuberculosis in resource-limited countries. ${ }^{10}$ This included patients who had at least 2 specimens (sputum, pleural aspirate, bronchial washings) sent for the diagnosis of suspected pulmonary TB and those with at least 1 sample sent for the diagnosis of extrapulmonary TB. By the above WHO specifications, ${ }^{10}$ patients were considered positive for TB if at least 1 out of 2 or more specimens sent for AFB microscopy was found positive for TB hence, patients who had only 1 sample sent for the diagnosis of suspected pulmonary TB were recruited only if this was positive for TB or from an extrapulmonary site.

The protocol for the diagnosis of pulmonary TB in $\mathrm{UCH}$ during the period the samples were obtained was to examine 3 sputum smear specimens, preferably early morning sputum specimens sent on consecutive days. The WHO requirement at the time was for at least 2 of these 3 specimens to be positive for AFB for a diagnosis of pulmonary TB to be made. It has however been shown by several studies that the incremental yield with the $3^{\text {rd }}$ smear is not significant enough to justify the increased workload resulting therefrom, and that 2 pulmonary-related specimens were sufficient for a diagnosis of TB. ${ }^{11,12}$

Patients with multiple specimens sent for the diagnosis of TB had further analysis of the results of only those specimens within the same presentation defined as specimens sent all within the period of 1 week. The demographic data and details about the results of AFB microscopy and culture were obtained and patients with incomplete records were excluded.

\section{Laboratory processes}

Specimens from sputum and other non-sterile sites were decontaminated with 5\% sodium hypochlorite solution (household bleach, JIK ${ }^{\mathrm{TM}}$, trademark Reckitt Benckiser) before centrifugation. Specimens from sterile sites such as cerebrospinal fluid were directly centrifuged and then subjected to Hot Ziehl-Neelsen (ZN) staining with $1 \%$ carbolfuchsin solution. ${ }^{13}$
Centrifugation was done at $3500 \mathrm{~g}$ with the CRU 5000 model centrifuge manufactured by IEC.

Examination of smears made from diagnostic specimens for acid fast bacilli (AFB) was done by 2 different medical laboratory scientists using binocular microscopes with oil immersion objectives. At least one length of a $20 \mathrm{~mm}$ long smear is examined carefully. This corresponds to 100 high power fields (HPF's) examined at 1000x magnification. ${ }^{11}$ Positivity on AFB microscopy was recorded as scanty or numerous AFB. These correspond to 1-9 AFB/1 length or $100 \mathrm{HPF}$ and $>10 \mathrm{AFB} / 1$ length or $100 \mathrm{HPF}$ respectively. Numerous AFB on microscopy corresponds to $1+, 2+$ or $3+$ positivity according to the IUATLD and WHO grading scales. ${ }^{11} 5 \%$ of the slides reviewed weekly were selected in a random but systematic manner for re-checking.

The processed specimens were then cultured directly onto Lowenstein-Jensen (L-J) culture medium ${ }^{6}$ and Pyruvic Acid Egg Medium (PAEM), a modification of the L-J medium in which glycerol is replaced by pyruvic acid to enhance the growth of $M$. Bovis ${ }^{13}$. Incubation was at $37^{\circ} \mathrm{C}$ for up to 8 weeks. The cultures were checked weekly for the growth of mycobacteria. The internal quality control measures used to confirm that the organism identified is mycobacterium tuberculosis include the reduction of nitrates to nitrites, the production of serpentine cords on smears from in-vitro grown colonies and the lack of pigment production in the dark or in the light. 5, 6

\section{Interpretation of results}

Detection of AFB in a sputum smear is not affirmative for $M$. tuberculosis because some non-tuberculous mycobacteria (NTM), otherwise known as atypical mycobacteria or mycobacteria other than tuberculosis (MOT'T) also cause pulmonary disease and may be found as the cause of disease or existing in supplying water as contaminants. ${ }^{7}$ Non-tuberculous mycobacteria, though less pathogenic than $M$. tuberculosis, can cause disease at various sites as well as disseminated disease in immunocompromised hosts. ${ }^{8}$ The recognition of atypical mycobacteria has significant implications for treatment in that first-line antituberculous agents are much less active against $M$. avium intercellulare (MAI), an atypical mycobacterial species, than against $M$. tuberculosis. In the 1997, American Thoracic Society (ATS) recommendations for MAI lung disease, clarithromycin replaces isoniazid and rifabutin is used in place of rifampicin. Therapy for pulmonary disease is usually given until culture has been negative for 12 months. ${ }^{8}$ Positive smear results, if the AFB found are $M$. tuberculosis, indicate that the patient 
is highly infectious. ${ }^{7}$ Negative smear results do not exclude the presence of less than 10,000 organisms / $\mathrm{ml}$ of sputum. AFB microscopy also tends to be negative in specimens from extra-pulmonary sites ${ }^{2}$. AFB microscopy is therefore, not conclusive for the diagnosis in case of positive findings, and it is not sensitive enough for exclusion of tuberculosis when the results are negative. ${ }^{7}$

The current "gold standard" for the diagnosis of tuberculosis is mycobacterial culture. Culture is about 500 times more sensitive than AFB microscopy and provides organisms for further investigations including drug susceptibility testing and genotyping. ${ }^{7}$

\section{Statistics}

The data collected were then analyzed using the Statistical Package for the Social Sciences (SPSS), Windows version 17.0.

\section{RESULTS}

A total of 694 specimens were received from 333 patients. There was 1 specimen each from 108 patients, 2 specimens each from 89 patients, and 3 specimens each from 136 patients. One hundred and twentynine $(38.7 \%)$ of these patients were found to be positive. 78 (60.5\%) were positive on AFB microscopy alone, $13(10.0 \%)$ on culture alone and 38 (29.5\%) on both culture and AFB microscopy. The actual age was stated for 87 of the cases positive for TB. The age range was 8 to 72 years with peak occurrence in the third decade of life.

The sex was recorded for all the positive cases. Sixtyeight $(68,52.7 \%)$ of these patients were females and $61(47.3 \%)$ were males giving a male to female ratio of 0.9 to 1 .

One hundred and sixteen of the 129 positive cases were positive by AFB microscopy while 13 patients were negative. Seventy-four $(63.8 \%)$ of them had scanty AFB, 42 of them $(36.2 \%)$ had numerous AFB. The 13 AFB-negative cases were found to be positive on culture thus, justifying their inclusion in the study for further assessment.

Fifty-one $(39.5 \%)$ of the 129 positive cases had positive culture results while $78(60.5 \%)$ had negative results. Out of the 51 cases which were found to be positive on culture, 38 cases $(74.5 \%)$ grew on Lowensen-Jensen culture medium alone, 10 (19.6\%) grew on Pyruvic Acid Enhanced Medium (PAEM) while 3 (5.9\%) grew on both culture media.

Smears showing numerous AFB were not more likely to show positive results on culture, smears with scanty AFB showed positive cultures in $37.8 \%$ while smears with numerous $\mathrm{AFB}$ on $\mathrm{ZN}$ staining showed positive cultures in $23.8 \%$ of cases.

When compared to culture, AFB microscopy was found to have a diagnostic specificity of $71.6 \%$ and a diagnostic sensitivity of $74.5 \%$.

\section{DISCUSSION}

In this study, a total of 13 cases were negative on AFB microscopy but positive on culture, this constituted $25.5 \%$ of the total of 51 cases which were positive on culture. A negative culture report on an AFB smear positive specimen may indicate the presence of noncultivable bacilli.

Seventy-eight (78) cases were negative on culture even though they had been found to be positive on AFB microscopy. This constituted $67.2 \%$ of the total of 116 cases which were positive on AFB microscopy. A negative culture report on AFB positive specimen is typical for patients undergoing successful therapy. ${ }^{7}$ With the recommended 6-month regimen, virtually all patients will have negative sputum cultures by the end of the third month. In some patients, especially those with cavitary disease and large numbers of organisms, AFB sputum conversion may follow culture conversion. This phenomenon is presumably due to the expectoration and microscopic visualization of dead bacilli. ${ }^{8}$

\begin{tabular}{llll}
\hline AFB MICROSCOPY & \multicolumn{3}{c}{ CULTURE } \\
\cline { 2 - 4 } & $\begin{array}{l}\text { POSITIVE } \\
\text { Number } \\
(\text { column } \%)\end{array}$ & $\begin{array}{l}\text { NEGATIVE } \\
\text { Number }\end{array}$ & $\begin{array}{l}\text { TOTAL } \\
\text { columber }\end{array}$ \\
& $28(54.9 \%)$ & $46(16.7 \%)$ & $74(22.7 \%)$ \\
Scanty AFB & $10(19.6 \%)$ & $32(11.6 \%)$ & $42(12.9 \%)$ \\
Numerous AFB & $13(25.5 \%)$ & $197(71.7 \%)$ & $210(64.4 \%)$ \\
Negative & 51 & 275 & 326 \\
TOTAL & & & \\
\hline
\end{tabular}

Table 1- Correlation of results of AFB microscopy with culture 
We did not find complete agreement in the results obtained from mycobacterial culture and AFB microscopy at the Tuberculosis Laboratory at UCH. We could not ascertain the specific causes of the disparities seen as this is a retrospective study. Some of the disparities found in the results of AFB microscopy and culture can be explained by laboratory errors. A previous study shows that false positive results on AFB microscopy may be due to clerical errors, serious microscope malfunction, grossly inadequate technique or inability to recognize acid fast bacilli due to inadequate training or high staff turnover. ${ }^{2}$ False negative results on AFB microscopy may suggest work overload, poor staining reagents or technique, inadequate microscopes, incorrect reading or carelessness. ${ }^{2}$ False negative results on culture may be due to long specimen transport time, the lack of sufficient technical efficiency, poor decontamination or culture preparation, use of expired or poorly prepared media, poor interpretation of findings and incorrect data recording. ${ }^{11}$ False positive results on culture have been described following crosscontamination in the laboratory. ${ }^{7}$

The percentages for positivity with AFB microscopy compared with culture obtained in this study may be compared with those from various parts of the world. ${ }^{14}$

Cases with numerous AFB on microscopy did not show an increased likelihood for yielding positive results on culture than those with scanty AFB. This is due to the fact that the recovery of AFB from microscopy is significantly affected by several factors including the adequacy of the specimen, the processes of collection, transport and decontamination of the specimen, the temperature of centrifugation and the amount of centrifugal force applied. ${ }^{7}$

The revised definition of a new sputum smear-positive pulmonary tuberculosis case is based on the presence of at least one acid fast bacillus (AFB + ) in at least one sputum sample in countries with a well functioning external quality assurance (EQA) system. ${ }^{15}$ Hence, both cases with scanty and numerous AFB on microscopy will be considered as pulmonary tuberculosis cases according to WHO specifications, provided there is an EQA program in the laboratory.

In resource-limited settings where tuberculosis is most prevalent, where facilities for mycobacterial culture is not readily available in most laboratories, and where the need to cut cost is paramount, it is possible to depend mostly upon AFB microscopy without the need for confirmation by culture for every index case. However, we are able to depend on AFB microscopy alone for diagnosis only to the extent that we are convinced that results therefrom are accurate. Culture may then be reserved for paediatric cases, cases of extra-pulmonary disease, sputum smear negative TB with HIV co-infection. ${ }^{3}$

The WHO new policy requires that a well functioning EQA system must be in place. In truth, this is the most important consideration because unless there is a process whereby we can ensure that each case diagnosed by AFB microscopy is truly positive and not a false positive case, there will be a continued need for culture to be done for confirmation. This means that in our resource-limited setting, our efforts should be directed most importantly towards assuring quality in the use of AFB microscopy.

In order to simplify and standardize EQA activities for AFB microscopy, and to prioritize EQA at National TB Control Programs (NTP), a practical EQA guideline was developed by an international working group and endorsed in 2002. These international EQA guidelines recommend three methods to evaluate laboratory performance: on-site assessment using a standardized questionnaire, panel testing of technician proficiency using centrally prepared slides, and blinded rechecking of a random sample of routine slides from each peripheral laboratory. ${ }^{2}$ These EQA processes for AFB microscopy have become operational in our laboratory at the UCH, Ibadan.

All the cases recruited into this study showed growth on culture within a period ranging between 3 and 8 weeks with an average duration period to growth of 4.8 weeks. Seven (7) cases were identified in the records which showed growth on culture within 1 week. These are not cases of tuberculosis as the confirmatory tests enumerated in the materials and methods section above were negative in these cases. Tuberculosis is caused by bacteria belonging to the Mycobacterium tuberculosis complex ( $M$ tuberculosis, $M$ bovis and $M$. africanum) which are slow-growing. Rapidly growing mycobacteria (RGM) are the Runyon group IV organisms that usually form colonies within 7 days of incubation as opposed to slow-growing mycobacteria, that is, Runyon groups I, II, and III and the $M$ tuberculosis complex group, that require longer incubation periods. RGM, though traditionally believed to be non-pathogenic or opportunistic species, have emerged as significant human pathogens, causing various infections in healthy and immunocompromised hosts. ${ }^{16}$ This underscores the importance of the accurate interpretation of findings on culture and the use of confirmatory tests. The recognition of RGM has significant implications for treatment purposes. These organisms are resistant to anti-tuberculous agents other than amikacin, but are 
variably susceptible to several other antibiotics, the most useful of which is clarithromycin. ${ }^{8}$

Both L-J and PAEM are able to support the growth of $M$ tuberculosis though growth is much better on $\mathrm{L}$ $\mathrm{J}$ medium than on PAEM. Our presumption is that the cases which grew on only L-J or both L-J and PAEM are likely cases of $M$. tuberculosis and those which grew on only PAEM are likely cases of $M$. bovis with a suggestion of a likely prevalence as high as $19.6 \%$. It is said that $M$. bovis has become rare, both in developed and developing countries. ${ }^{5,8}$ There is need for the speciation of subsequent isolates to determine the particular strain of mycobacteria causing disease in each case. Speciation can be done by the use of biochemical tests, liquid media with radiometric growth detection, nucleic acid probes and High Perfomance Liquid Chromatography (HPLC) of mycolic acids. ${ }^{8}$

AFB microscopy is shown in this study to have a diagnostic specificity of $71.6 \%$ and a sensitivity of $74.5 \%$. Previous studies from other developing countries showed higher specificity and sensitivity values for AFB microscopy. Shea et al ${ }^{17}$ showed a specificity of $100 \%$ and a sensitivity of $93.1 \%$ in Uganda while Farnia et al ${ }^{18}$ showed a specificity of $96 \%$ and a sensitivity of $78 \%$ in Iran. These results were obtained with similar procedures.

Modification of laboratory processes, ${ }^{2}$ re-training of personnel, supply of new microscopes, and the implementation of EQA programs have been shown to significantly increase the accuracy of TB diagnosis.

\section{CONCLUSION}

An important first step in the microbiological diagnosis of tuberculosis is the clinician's suspicion and the requisition of investigations.

In general, there is need for training and re-training (continuing education) of medical laboratory scientists to improve smear and culture preparation, staining and reading techniques as well as the provision and the maintenance of necessary equipment such as microscopes.

If AFB microscopy is to be done routinely without confirmation by culture in our resource-limited setting, then the overriding need is for quality to be fully assured in its use.

\section{Acknowledgements}

The authors acknowledge the contributions of Mr F.A. Obaseki and Mr A.O. Adebiyi, both medical laboratory scientists for their technical assistance.
1. Wambani SJ \& Reeve P. Tuberculosis. In The Imaging of Tropical Diseases, with Epidemiological, Pathological and Clinical Correlation. 2nd Edition. (ed. Palmer P. and Reeder M.) Springer -Verlag , USA. 2001; Chapter 5.

2. Van Rie A, Fitzgerald D, Kabuya G, et al (2008) Sputum smear microscopy: evaluation of the impact of training, microscope distribution, and the use of External Quality Assessment Guidelines for resource poor settings. J. Clin. Microbiol. doi:10.1128/JCM.01553-07.

3. World Health Organization (WHO). Global Tuberculosis Control: Country Profile for Nigeria, WHO report 2008; 129-132 C) World Health Organization, Geneva.

4. World Health Organization (WHO) The Stop Tuberculosis strategy, pages 3-5 (C) World Health Organization, Geneva. 2006

5. Brooks GF, Butel JS, Morse SA. Mycobacteria. In Jawetr, Melnick, \& Adelberg's Medical Microbiology. $23^{\text {rd }}$ edn. (ed. G.F. Brooks, J.S. Butel, S.A. Morse)The McGraw-Hill Companies Inc., Asia. 2004; Chapter 24, 319- 326.

6. Cheesbrough M. Mycobacterium tuberculosis. In District Laboratory Practice in Tropical Countries (Part 2). $1^{\text {st }}$ edn. (ed M. Cheesbrough) Cambridge University Press, India. 2000; 209.

7. Zwolska Z Modern microbiological diagnostic methods of tuberculosis in clinical practice, 2005; 70-71.

8. Raviglione M.C. \& O'Brien R.J. Section 8 Mycobacterial diseases. Chapters 168 (Antimycobacterial agents) \& 169 (Tuberculosis). In Harrison's Principles of Internal Medicine. $15^{\text {th }}$ edn. (ed. Braunwald E, Fauci A.S, Kasper D.L., Hauser S.L., Longo D.L., Jameson J.L.) McGraw-Hill Companies, Inc, USA. 2001; 1017-1034.

9. Osho J.A. Treatment of opportunistic infections of HIV with reference to tuberculosis management (powerpoint presentation) 2006. Available at http://www.nelanigeria.org/otherpages / conferences-recent1.html.

10. Improving the diagnosis and treatment of smearnegative pulmonary and extrapulmonary tuberculosis among adults and adolescents. Recommendations for HIV-prevalent and resource-constrained settings. Stop TB Department, Department of HIV/AIDS, World Health Organisation. WHO, Geneva, Switzerland, 2006, available at http:/ / www.who.int/entity/tb/ publications $/ 2006 /$ tbhiv recommendations.pdf

11. Reider HL, Van Deun A, Kam KM, et al: Table II.3. Grading scales for bright field (Ziehl-Neelsen) and fluorescence microscopy, Priorities for Tuberculosis Bacteriology Services in Low- 
income countries. International Union Against Tuberculosis and Lung Disease $2^{\text {nd }}$ Edition 2007.

12. Improving the Diagnosis of Tuberculosis through Optimization of Sputum Microscopy. Expert Consultation. World Health Organization CWHO 2005

13. Ziehl Neelsen staining: International Union against Tuberculosis (IUAT), Technical Guide for sputum Examination for Tuberculosis by Direct Microscopy. Bulletin of the International Union Against Tuberculosis, supplement No.2, 1978.

14. Ridderhof J: AFB smears for diagnosis and monitoring treatment . (C) Division of laboratory systems, Public Health Practice Program Office, Centers for Disease Control and Prevention. Available at http://wwwn.cdc.gov/dls/ila/cd/ india/Jan22.
15. World Health Organization (WHO) New WHO policies: Definition of a new sputum smearpositive tuberculosis case, 2009; 2 C World Health Organization, Geneva.

16. Xiang YH, Indra DE, Kalen LJ. Rapidly Growing Mycobacteria: Clinical and Microbiologic Studies of 115 Cases American Journal of Clinical Pathology. 2007; 128 (4), 612-621.

17. Shea YR, Davis JL, Huang L, et al: High Sensitivity and Specificity of Acid-Fast Microscopy for Diagnosis of Pulmonary Tuberculosis in an African Population with a High Prevalence of Human Immunodeficiency Virus_ J Clin Microbiol, May 2009, 1553-1555 Vol. 47, No. 5.

18. Farnia P, Mohammadi F, Zarifi Z, et al: Improving Sensitivity of Direct Microscopy for Detection of Acid-Fast Bacilli in Sputum: Use of Chitin in Mucus Digestion J Clin Microbiol. 2002 February; 40(2): 508-511. 